Bol. Soc. Bot. México 57: 37-48 (1995)

\title{
Los agaves de las Antillas
}

\author{
ALBERTO ÁLVAREZ DE ZAYAS \\ Instituto de Ecología y Sistemática. Centro Nacional de Biodiversidad. Carretera de Varona Km 3.5, \\ Capdevila, Boyeros, Ciudad Habana, Cuba.
}

\begin{abstract}
Resumen.Se brinda una lista actualizada de los 43 taxa antillanos de Agave, más nueve que solo se conocen de cultivo. Se hace un análisis comparativo de las principales especies utilizando los caracteres morfológicos más relevantes, esto permite separar 12 especies nativas que son lo suficientemente diferentes y representativas, en torno a las cuales y en estrecha relación con ellas se pudieron reunir las restantes. Se discuten algunos argumentos sobre las relaciones entre las especies antillanas y sus parientes continentales. Se proporcionan esquemas ilustrativos,tablas y un mapa con la distribución de los taxa.

Palabras clave: Agavaceae, Agave, Antillas, morfología.

Abstract.In this paper an updated list of the 43 Caribbean taxa of Agave, plus nine taxa only know as cultivated plants is given. A comparative study of the main species is done using their most important morphological characters; this allows to separate 12 native species that are different and representative, in such a way that is possible to join the rest of the related species. Aspects of the relationships among the Caribbean species and their continental relatives are discussed. Illustrative drawings, tables and a map with the distribution of the taxa are also given.
\end{abstract}

Key words. Agavaceae, Agave, Antilles; morphology.

\section{INTRODUCCIÓN}

La familia Agavaceae está presente en la Antillas con los géneros Agave L. y Furcraea Vent., plantas que participan en muchas de las variadas formaciones vegetales de estos territorios insulares. Se considera el territorio antillano formado por las Bahamas, Antillas Mayores, Antillas Menores, incluidas las Antillas Holandesas y territorios insulares adyacentes.

Estas plantas americanas formaron parte de lo que los europeos calificaban en sus sorprendentes relatos como "maravillas del Nuevo Mundo"; fueron relativamente minuciosas las descripciones que de ellas hicieron y que aparecen en los diarios de viajeros y cronistas.

Las voces taínas "maguey", "heniquén" y "cabuya" son algunas con las que se designan especies de Agave y que fueron oídas por primera vez en las Antillas, más tarde acompañaron a los colonizadores en sus viajes hacia el continente, difundiéndolas.

Los usos que los indios antillanos hacían de estas plantas fueron realmente limitados si se comparan con el formidable espectáculo que aún brindan las culturas mesoamericanas, donde los magueyes han tenido y tienen tan variados usos, por lo que están profundamente ligados a la subsistencia y costumbres de esos pueblos. La confección de hilos y tejidos diversos a partir de las hojas de los magueyes sigue siendo muy importante entre los pueblos de México, pero fue siempre más reducida entre los aruacos de las Antillas, ellos preferían las fibras de las cortezas de los árboles para algunos de esos menesteres. Sin embargo, parece más antillana la costumbre de emborrachar a los peces de río con el jugo de las hojas machacadas de los magueyes para facilitar su captura.

Desde el punto de vista fitogeográfico resulta de interés que, Agave, un género con más de 250 especies en el continente, posea una representación tan significativa con cerca de 50 especies en tierras antillanas; esta interesante cuestión fue analizada por Álvarez (1989) y discutida también por García-Mendoza (1995). En este artículo se pretende no sólo brindar una lista actualizada de los taxa en las Antillas, sino también aproximarse a una interpretación de las relaciones entre las especies de ésta región a través de los aún escasos datos disponibles, para facilitar de algún modo, una hipótesis sobre los posibles parientes continentales de estas especies.

\section{Aportes al conocimiento de las especies antillanas de Agave}

Los agaves antillanos son relativamente abundantes y sobre todo muy variables en sus caracteres morfológicos. Las especies conocidas hasta el siglo pasado eran pocas y en general reflejan la escasez de colectas en esa época. Las especies descritas en los siglos XVIII y XIX, fueron: $A$. vivipara L.(1753), A. karatto Mill.(1768), A. antillarum Descourt.(1827), A. sobolifera Salm-Dyck (1834), A. offoyana 
Jacobi (1864), A. legrelliana Jacobi (1865), A willdingii Tod. (1886) y A. morrisii Baker (1887).

En el siglo XX, Trelease (1913), produce la primera recopilación y el primer estudio integral de Agave en esta región, su trabajo fue meticuloso y exhaustivo en la revisión bibliográfica y en la consulta de las colecciones de los herbarios conocidos hasta esa fecha.

Desde finales del pasado siglo el interés de ampliar las colecciones de especímenes antillanos por instituciones norteamericanas y europeas era muy grande y una vez concluídas las luchas independentistas en Cuba y Puerto Rico se organizaron varias expediciones como las de Britton y Shafer del New York Botanical Garden, Trelease, Brace y Harris del Missouri Botanical Garden, Boldingh del herbario de Utrecht y Ekman que colectaba para Urban de Berlín.

Trelease elevó el número de especies hasta 50 con prácticamente un $100 \%$ de endemismos para las especies en cada isla, la concepción de las especies de Trelease es muy peculiar pues cada variación merecía un nombre, este criterio no era tan marcado allí donde se disponía de abundantes materiales, pero la mayoría de sus especies fueron fundadas en uno o pocos especímenes.

La taxonomía supraespecífica no fue precisa y como se estilaba en la escuela botánica americana de aquella época, las relaciones entre las especies fueron reflejadas como "grupos"; dos años después Berger (1915) elevó a la categoría de Secciones de Agave a dichos grupos.

Cuando Trelease se traslada a Europa encuentra en los herbarios de Ekman algunos materiales de Agave que no duda en describir como nuevas especies o variedades. Lógicamente el tabajo de Trelease merece revisión, pero ésta difícil tarea requiere mesura y consideración, pues no parecen adecuados trabajos como los de Hummelinck $(1936,1938)$, quien al revisar los agaves de la sección Viviparae agregó más especies a la ya abigarrada lista legada por Trelease para las pequeñas islas al norte de América del Sur.

Por otro lado, contribuciones como las de Gillis (1976) para Bahamas, reducen a una sola, las seis especies descritas por Trelease, con el simple argumento de que la mayoría de ellas eran conocidas solamente del material tipo y $\sin$ que se reportaran nuevas colectas y estudios comparativos, es tambien inadecuado, pero en el otro extremo.

La revisión de las Agavaceae cubanas y en particular de Agave, (Álvarez, 1980a, 1980b, 1985), resultó una compleja labor que ha requerido primeramente disponer de suficiente material de herbario. Hasta 1974 los herbarios cubanos contenían unos 50 especímenes de Agave, pero cuentan ya hoy con más de 500 y aún así muchas y enigmáticas localidades se conocen sólo a través de materiales estériles.

\section{CARACTERÍSTICAS GENERALES DE LOS AGAVES ANTILLANOS}

Los estudios en las especies cubanas y la revisión de herbarios y descripciones permiten reconocer, al menos de modo provisional, los patrones morfológicos enlistados en el Cuadro 1. Los caracteres vegetativos y reproductivos se esquematizan en las Figuras 1-5. Es conveniente resaltar que, el tamaño forma y curvatura de los dientes (Fig. 2q), es cambiante. Las ramas de la panícula y las siluetas de las inflorescencias son consideradas de importancia (Fig. 3). Las flores son variables aún en la misma población, pero todas parecen seguir un mismo patrón morfológico con pocas variaciones en la profundidad del tubo (Fig. 4d,e), tépalos (Fig. 4f,g), pedicelos (Fig. 4h,l) y en la inserción de los filamentos. Así mismo, los frutos presentan variaciones en sus bases y ápices (Fig. $5 \mathrm{e}-\mathrm{j}$ ), pero su forma general merece la mayor atención (Fig. 5a-d).

Los hijuelos o bulbilos son similares (Fig. 5k-m), pero su presencia como principal forma de reproducción está confinada a la Sección Caribaeae y Viviparae; la eventualidad de ellos en Antillanae y Bahamanae parece indicar que el desarrollo y migración de esas especies debió ocurrir en las Antillas de oeste a este.

Otro de los caracteres que se ha empleado en la delimitación de las especies de Agave ha sido la estructura anatómica de los complejos estomáticos de la epidermis foliar. Desde finales del siglo pasado se han realizado estudios en este sentido y hoy en dia se sabe que con independencia de las variaciones, hay patrones morfológicos que permiten reconocer especies o grupos afines de especies (Blunden y Jewers, 1973; Álvarez, 1990). Álvarez (1979), en el caso particular de los agaves cubanos, logra delimitar las especies de la región occidental del país.

\section{VARIABILIDAD Y ESPECIACIÓN}

Un análisis somero de las especies antillanas de Agave permite separar 12 especies suficientemente diferentes y representativas, en torno a las cuales y en estrecha relación con ellas se pudieran reunir las restantes. Esto no quiere decir que se desconoce la validéz o la existencia de las demás, sino que las especies seleccionadas son representativas de líneas de evolución del género en la región.

En el Cuadro 2 se muestra la comparación de las 12 especies seleccionadas utilizando los 17 caracteres morfológicos sumarizados en el Cuadro 1. A partir de la comparación elemental de las semejanzas y diferencias podemos inferir cuales son las relaciones de afinidad entre las especies y a través de ello aproximarnos a la comprensión de la especiación de los agaves en las Antillas. Los resultados de este análisis se presentan en el fenograma correspondiente (Fig. 6).

Las especies 2-7 (Cuadro 2), de la sección Antillanae muestran la variabilidad de ese grupo de amplia distribución en las Antillas Mayores; la relación de A. sobolifera (4) y A. missionum (5) con A. bahamana (1) de la sección Bahamanae demuestra antiguas relaciones entre esas especies muy distantes y aisladas geográficamente. (Fig. 7).

A. offoyana (2), A. antillarum (3) y A.portoricensis(6) parecen haber seguido líneas de especiación diferentes en territorios separados desde el Mioceno (Gentry, 1982). A. cajalbanensis (7), se muestra a mitad de camino entre las 
CuAdro 1. Lista de caracteres y sus estados.

\section{Vegetativos}

Reproductivos

1 - Dimensiones de las plantas:

a-plantas grandes, con más de $200 \mathrm{~cm}$ de diámetro (Fig. 1b,c).

b-plantas moderadas, $110-180 \mathrm{~cm}$ de diámetro.

c-plantas pequeñas, menores de $100 \mathrm{~cm}$ de diámetro (Fig. 1a,d,e).

2 - Desarrollo de estolones con hijuelos basales:

a-plantas eventual o frecuentemente cespitosas (Fig. 1e).

b-plantas nunca estoloníferas (Fig. 1a-d).

3 - Desarrollo del tallo:

a-plantas acaules.(Fig. 1b-e).

b-plantas subcaulescentes, tallos de 30-50 cm, rara vez más (Fig. 1a).

4 - Cantidad de hojas en la roseta:

a-plantas con más de 70 hojas (Fig. 1b,c).

b-moderada cantidad de hojas, 40-60 (Fig. 1a,d).

c-pocas hojas, menos de 40 (Fig. le).

5 - Disposición de las hojas en la roseta:

a-hojas rectas o casi rectas (Fig. 1d,e).

b-hojas algo flexuosas (Fig. 1b).

c-hojas muy flexuosas (Fig. 1a).

6 - Color de las hojas:

a-grises a glaucas. b-verde grisáceas. c-verdes d-verde claras.

7 - Forma de las hojas:

a-lanceoladas (Fig. 2a).b-anchamente lanceoladas (Fig. 2c,d).

c-estrechamente lanceoladas (Fig. 2b).

8 - Concavidad de las hojas:

a-cóncavas (Fig. 2a,b). b-casi planas (Fig. 2c,d).

9 - Espina terminal:

a-aguda, canalículo moderado (Fig. 2h-k).

b-muy aguda hasta acicular, canalículo breve (Fig. 2l-m).

c-obtusa a roma, canalículo muy largo (Fig. 2e-g).

10 - Dientes del margen de la hoja y su separación:

a-dientes bien desarrollados y subdistantes (Fig. 2n-o),

b-dientes diminutos y muy próximos (Fig. 2p).
11 - Forma de la panícula:

a-oblongo-piramidales (Fig. 3a).

b-oblongas (Fig. 3b).

c-oblongo-fusiformes (Fig. 3c).

12 - Desarrollo relativo del pedúnculo floral:

a-breve (Fig. 3d).

b-moderado (Fig. 3e).

c-alargado (Fig. 3f).

13 - Ramas de la panícula: a-bien desarrolladas y tripartidas (Fig. 3g).

b-algo compactas y trilobadas (Fig. $3 \mathrm{~h}$ ).

c-muy compactas y unilobadas (Fig. 3i).

14 - Color de las flores:

a-amarillo verdosas.

b-amarillas.

c-doradas a anaranjadas.

15 - Tamaño de las flores:

a-grandes, más de $60 \mathrm{~mm}$ (Fig. 4a).

b-medianas, de 40-55 mm (Fig. 4b).

c-pequeñas, menores de $35 \mathrm{~mm}$ (Fig. 4c).

16 - Forma de los frutos:

a-globosos a subesféricos (Fig. 5a).

b-oblongos (Fig. 5b,d).

c-subcilíndricos (Fig. 5c).

17 - Desarrollo de hijuelos del escapo o bulbilos: a-nunca producen bulbilos.

b-eventualmente producen bulbilos.

c-frecuentemente producen bulbilos.

CuAdro 2. Matriz de comparación de los caracteres de 12 especies representativas, de las 43 analizadas. Los caracteres y sus estados se mencionan con números y letras según el Cuadro 1.

Caracteres morfológicos

\begin{tabular}{llllllllllllllllll}
\hline Especies de Agave & $\mathrm{1}$ & 2 & 3 & 4 & 5 & 6 & 7 & 8 & 9 & 10 & 11 & 12 & 13 & 14 & 15 & 16 & 17 \\
1. A. bahamana & $\mathrm{a}$ & $\mathrm{b}$ & $\mathrm{a}$ & $\mathrm{a}$ & $\mathrm{b}$ & $\mathrm{b}$ & $\mathrm{a}$ & $\mathrm{a}$ & $\mathrm{a}$ & $\mathrm{ab}$ & $\mathrm{b}$ & $\mathrm{a}$ & $\mathrm{a}$ & $\mathrm{b}$ & $\mathrm{b}$ & $\mathrm{c}$ & $\mathrm{b}$ \\
2. A. offoyana & $\mathrm{a}$ & $\mathrm{b}$ & $\mathrm{a}$ & $\mathrm{a}$ & $\mathrm{c}$ & $\mathrm{c}$ & $\mathrm{a}$ & $\mathrm{a}$ & $\mathrm{a}$ & $\mathrm{a}$ & $\mathrm{a}$ & $\mathrm{c}$ & $\mathrm{a}$ & $\mathrm{c}$ & $\mathrm{a}$ & $\mathrm{b}$ & $\mathrm{b}$ \\
3. A. antillarum & $\mathrm{b}$ & $\mathrm{b}$ & $\mathrm{a}$ & $\mathrm{b}$ & $\mathrm{c}$ & $\mathrm{d}$ & $\mathrm{a}$ & $\mathrm{a}$ & $\mathrm{a}$ & $\mathrm{a}$ & $\mathrm{b}$ & $\mathrm{a}$ & $\mathrm{c}$ & $\mathrm{c}$ & $\mathrm{b}$ & $\mathrm{c}$ & $\mathrm{b}$ \\
4. A. sobolifera & $\mathrm{a}$ & $\mathrm{b}$ & $\mathrm{ab}$ & $\mathrm{a}$ & $\mathrm{c}$ & $\mathrm{c}$ & $\mathrm{a}$ & $\mathrm{a}$ & $\mathrm{a}$ & $\mathrm{a}$ & $\mathrm{b}$ & $\mathrm{a}$ & $\mathrm{a}$ & $\mathrm{b}$ & $\mathrm{b}$ & $\mathrm{c}$ & $\mathrm{b}$ \\
5. A. missionum & $\mathrm{a}$ & $\mathrm{b}$ & $\mathrm{a}$ & $\mathrm{a}$ & $\mathrm{c}$ & $\mathrm{c}$ & $\mathrm{b}$ & $\mathrm{a}$ & $\mathrm{a}$ & $\mathrm{a}$ & $\mathrm{b}$ & $\mathrm{b}$ & $\mathrm{a}$ & $\mathrm{b}$ & $\mathrm{b}$ & $\mathrm{c}$ & $\mathrm{b}$ \\
6. A. portoricensis & $\mathrm{a}$ & $\mathrm{b}$ & $\mathrm{a}$ & $\mathrm{a}$ & $\mathrm{c}$ & $\mathrm{c}$ & $\mathrm{a}$ & $\mathrm{a}$ & $\mathrm{a}$ & $\mathrm{a}$ & $\mathrm{c}$ & $\mathrm{a}$ & $\mathrm{b}$ & $\mathrm{a}$ & $\mathrm{b}$ & $\mathrm{a}$ & $\mathrm{b}$ \\
7. A. cajalbanensis & $\mathrm{b}$ & $\mathrm{b}$ & $\mathrm{a}$ & $\mathrm{b}$ & $\mathrm{b}$ & $\mathrm{a}$ & $\mathrm{b}$ & $\mathrm{b}$ & $\mathrm{a}$ & $\mathrm{a}$ & $\mathrm{b}$ & $\mathrm{b}$ & $\mathrm{a}$ & $\mathrm{c}$ & $\mathrm{b}$ & $\mathrm{c}$ & $\mathrm{a}$ \\
8. A. tubulata & $\mathrm{c}$ & $\mathrm{b}$ & $\mathrm{a}$ & $\mathrm{b}$ & $\mathrm{a}$ & $\mathrm{d}$ & $\mathrm{b}$ & $\mathrm{b}$ & $\mathrm{b}$ & $\mathrm{a}$ & $\mathrm{c}$ & $\mathrm{c}$ & $\mathrm{c}$ & $\mathrm{a}$ & $\mathrm{c}$ & $\mathrm{b}$ & $\mathrm{a}$ \\
9. A. albescens & $\mathrm{c}$ & $\mathrm{b}$ & $\mathrm{a}$ & $\mathrm{b}$ & $\mathrm{a}$ & $\mathrm{a}$ & $\mathrm{b}$ & $\mathrm{b}$ & $\mathrm{a}$ & $\mathrm{a}$ & $\mathrm{c}$ & $\mathrm{c}$ & $\mathrm{c}$ & $\mathrm{a}$ & $\mathrm{c}$ & $\mathrm{c}$ & $\mathrm{a}$ \\
10. A. nashii & $\mathrm{c}$ & $\mathrm{a}$ & $\mathrm{a}$ & $\mathrm{c}$ & $\mathrm{a}$ & $\mathrm{a}$ & $\mathrm{c}$ & $\mathrm{a}$ & $\mathrm{a}$ & $\mathrm{b}$ & $\mathrm{b}$ & $\mathrm{a}$ & $\mathrm{c}$ & $\mathrm{a}$ & $\mathrm{c}$ & $\mathrm{c}$ & $\mathrm{a}$ \\
11. A. karatto & $\mathrm{a}$ & $\mathrm{b}$ & $\mathrm{a}$ & $\mathrm{a}$ & $\mathrm{b}$ & $\mathrm{c}$ & $\mathrm{a}$ & $\mathrm{a}$ & $\mathrm{c}$ & $\mathrm{b}$ & $\mathrm{b}$ & $\mathrm{a}$ & $\mathrm{b}$ & $\mathrm{b}$ & $\mathrm{b}$ & $\mathrm{c}$ & $\mathrm{c}$ \\
12. A. vivipara & $\mathrm{c}$ & $\mathrm{ab}$ & $\mathrm{b}$ & $\mathrm{b}$ & $\mathrm{c}$ & $\mathrm{b}$ & $\mathrm{b}$ & $\mathrm{a}$ & $\mathrm{b}$ & $\mathrm{b}$ & $\mathrm{b}$ & $\mathrm{a}$ & $\mathrm{b}$ & $\mathrm{b}$ & $\mathrm{b}$ & $\mathrm{c}$ & $\mathrm{c}$ \\
\hline
\end{tabular}


especies de las secciones Antillanae y las de Antillares aquí representadas por A. albescens (8) y A. tubulata (9).

A. nashii (10), tal y como Trelease (1913) había planteado, muestra afinidad con las especies de la sección Antillares y en particular con $A$. albescens, no muy distante geográficamente; aún así $A$. nashii y probablemente $A$. inaguensis (sección Inaguenses) han seguido una evolución muy peculiar entre los agaves de las Antillas.

Otra relación interesante es la de A. karatto (11) de la sección Caribaeae con algunas especies de la sección Antillanae, pero evidentemente son más los caracteres que la aproximan a A. vivipara (12) de la sección Viviparae.

El desarrollo de hijuelos del escapo o bulbilos se presenta de manera eventual en muchas de las especies de las secciones Antillanae y Bahamanae, sin embargo constituye la forma principal de reproducción en las especies de Caribaeae y Viviparae. Se puede suponer que este carácter ha migrado desde las Antillas Mayores o Bahamas hacia las Antillas Menores y Holandesas.

De acuerdo con las inferencias de las posibles vías migratorias del género Agave en esta región, formuladas por el autor (Álvarez, 1989) y por García-Mendoza (1995)es de suponer, con los resultados ahora disponibles que el centro de evolución de Agave debió estar en las Antillas Mayores donde ahora es posible encontrar la mayor diversidad de especies en la región y específicamente en Cuba.

Las especies de Caribaeae y Viviparae son tan variables que en muchos casos más parecen tratarse de clones que de propias especies. El proceso de clonación realmente pudo ocurrir pues el flujo migratorio de los indios Caribes entre las Bahamas y las Antillas Menores, como señala en su obra el cronista Labat (1722), pudo haber introducido y diseminado algunas de las formas colectadas en Bahamas hacia otras islas del Caribe y las costas norteñas de Venezuela, donde eran empleadas en la confección de cordeles para la pesca y en otros menesteres.

En cuanto al discutido tema de las relaciones de las especies antillanas con las del continente, se puede reafirmar que las especies yucatecas no guardan relación con las antillanas y que tal vez las especies mesoamericanas serían las hermanas comunes de aquellas formas originales que migrando desde el continente norte pasaron a las protoantillas (Gentry, 1982; Álvarez, 1989).

Algunas especies de Agave de Guatemala recuerdan por sus inflorescencias laxas con flores amarillo verdosas, compactas en el extremo de las ramas y las hojas más bien pequeñas, a las especies de la sección Antillares. Por ejemplo, A. albescens se asemeja a algunas de las especies descritas por Trelease (1915) y recopiladas por GarcíaMendoza y Lott (1994).

Los agaves de la sección Antillares ocupan precisamente los afloramientos cubanos y posiblemente antillanos más antiguos aparecidos desde el Eoceno y que se ubican en el occidente, centro y extremo oriental de la isla de Cuba.

Para dilucidar esta importante cuestión y llegar a comprender las relaciones filogenéticas de las especies de
Agave en la Región Caribe del Reino Neotropical harán falta estudios comparativos más profundos.

\section{ESPECIES RECONOCIDAS DE AGAVE PARA LAS ANTILLAS Y COSTA NORTE DE SUDAMÉRICA}

\section{Sección Bahamanae}

Plantas grandes, no cespitosas, acaulescentes. Hojas de 100$300 \times 15-25 \mathrm{~cm}$, numerosas, flexuosas y cóncavas, lanceoladas, verde grisáceas; espina fuerte, decurrente; dientes variables. Inflorescencias grandes con panículas amplias en el tercio superior, oblongas. Flores amarillas de 45-60 mm. Cápsulas oblongas, variables. Eventualmente bulbíferas.

1) A. bahamana Trel., Mem. Nat. Acad. Sci. 11: 40. 1913. A. acklinicola Trel., Mem. Nat. Acad. Sci. 11: 41. 1913. A. braceana Trel., Mem. Nat. Acad. Sci. 11:40. 1913. A. cacozela Trel., Mem. Nat. Acad. Sci. 11: 42. 1913. A. indagatorum Trel., Mem. Nat. Acad. Sci. 11:42. 1913. A. millspaughii Trel., Mem. Nat. Acad. Sci. 11: 41. 1913.

Habitan en las islas Bahamas, en matorrales costeros, sobre suelos calizos, casi a nivel del mar.

Gillis (1967), revisó someramente los materiales estudiados por Trelease y redujo las 6 especies a una sola. Con independencia de la variabilidad de las especies y del parecido que existe entre ellas, algunas variaciones notables como los frutos estrechamente oblongos de A.indagatorum, las espinas diminutas de A. acklinicola y A. indagatorum y las flores pequeñas de $A$. braceana carecieron de un análisis adecuado. Por lo que aún esta sección necesita estudios para delimitar bien los taxa y aclarar sus relaciones.

\section{Sección Inaguenses}

Plantas pequeñas, cespitosas, acaulescentes. Hojas de $30-60 \times 3-9 \mathrm{~cm}$, pocas en la roseta, erectas, rígidas, glaucas; espina moderada, decurrente; dientes diminutos muy próximos. Inflorescencias pequeñas, laxas, con pocas ramas. Flores de 35-50 mm, amarillo-verdosas, compactas al final de las ramas. Cápsulas oblongas, pequeñas. No bulbíferas.

2) A. nashii Trel., Mem. Nat. Acad. Sci. 11: 45-46. 1913.

3) A. inaguensis Trel., Mem. Nat. Acad. Sci. 11: 47. 1913.

Crecen en las Bahamas suroccidentales en matorrales costeros secos y a veces arenosos casi a nivel del mar.

Las hojas glaucas, estrechas, acanaladas y pequeñas, las inflorescencias laxas, pequeñas y de flores amarillo verdosas compactas y el desarrollo de hijuelos estoloníferos imprimen a este grupo una singularidad notable entre las especies antillanas.

Este grupo tiene caracteres en común con algunos agaves de Bahamas, como son los diminutos dientes foliares de A. indagatorum y A. acklinicola, el color de las hojas y el aspecto de la inflorescencia de A. albescens de Cuba oriental. 


\section{Sección Antillares}

Plantas medianas a pequeñas, no cespitosas, acaulescentes. Hojas de 40-100 x 8-12 (-20) cm, en su mayoría anchamente lanceoladas, verde claras a glaucas; espina moderada, decurrente; dientes bien desarrollados. Inflorescencias laxas, panículas estrechamente oblongas casi desde la base. Flores amarilloverdosas o amarillas, agregadas en el extremo de las ramas. Cápsulas oblongas. Nunca bulbíferas.

4) A. tubulata Trel., Mem. Nat. Acad. Sci. 11: 45. 1913.

5) A. tubulata Trel., ssp. brevituba A. Álvarez. Revista Jard. Bot. Nac. Cuba 5(3): 3-16. 1985.

A. ekmanii Trel., Feddes Repert. 22: 374. 1926.

6) A. papyrocarpa Trel., Mem. Nat. Acad. Sci. 11: 44. 1913.

7) A. papyrocarpa Trel. ssp. macrocarpa A. Álvarez, Revista Jard. Bot. Nac. Cuba 5(3): 3-16. 1984.

8) A. jarucoensis A. Álvarez, Revista Jard. Bot. Nac. Cuba 1(1): 5-11. 1980.

9) A. brittoniana Trel., Mem. Nat. Acad. Sci. 11: 44-45. 1913. 10) A. brittoniana Trel., ssp. brachypus (Trel.) A. Álvarez (en prensa).

11) A. brittoniana Trel., ssp. spirituana A. Álvarez (en prensa). 12) A. acicularis Trel., Mem. Nat. Acad. Sci. 11: 34. 1913. 13) A. grisea Trel., Mem. Nat. Acad. Sci. 11: 34-35. 1913. 14) A. albescens Trel., Mem. Nat. Acad. Sci. 11: 44. 1913.

Habitan la isla de Cuba e isla de la Juventud (Isla de Pinos) en matorrales xeromorfos sobre calizas y sobre rocas ultrabásicas.

Nuevas localidades de agaves para esta sección han sido descubiertas en los últimos años y su estudio permitirá aclarar las relaciones entre las especies de la misma y los de la sección Antillanae.

A. willdingii Todaro (Hort. Bot. Panorm. 2:3. 1886), descrito a partir de plantas en cultivo en Europa de procedencia desconocida, fue incluida por Trelease en esta sección, pero el color anaranjado de sus flores la hacen más cercana a las especies que como $A$. antillarum crecen en La Española.

\section{Sección Antillanae}

Plantas grandes, no cespitosas, mayormente acaulescentes. Hojas de 100-300 x 12-20 cm, flexuosas, cóncavas, lanceoladas, verdes; espina bien desarollada; dientes grandes y subdistantes. Inflorescencias grandes, panículas oblongas a piramidales desde la base o más arriba. Flores de 50-60 mm o más, amarillas a anaranjadas. Cápsulas oblongas a subcilíndricas. Eventualmente bulbíferas.

15) A. antillarum Descourt., Fl. Pitt. et Med. Antilles 4: 239. 1827.

16) A. brevipetala Trel., Feddes Repert. 23: 362. 1927.

17) A. brevispina Trel., Feddes Repert. 23: 363. 1927.

18) A. intermixta Trel., Mem. Nat. Acad. Sci. 11: 32. 1913.

19) A. sobolifera Salm-Dyck, Hort. Dyck. 8: 307. 1834. A. morrisii Baker, Gard. Chron. 3(1): 543-549. 1887.

20) A. harrisi Trel., Mem. Nat. Acad. Sci. 11: 34. 1913.

21) A. longipes Trel., Mem. Nat. Acad. Sci. 11: 36. 1913.

22) A. missionum Trel., Mem. Nat. Acad. Sci. 11: 37. 1913.
A. eggersiana Trel., Mem. Nat Acad. Sci. 11: 28. 1913. 23) A. offoyana Jacobi, Hamburg. Gart. Blumenzeit. 501. 1864. A. legrelliana Jacobi, Hamburg. Gart. Blumenzeit. 507. 1865.

24) A. anomala Trel., Mem. Nat. Acad. Sci. 11: 36. 1913.

25) A. shaferi Trel., Mem. Nat. Acad. Sci. 11: 35. 1913.

26) A. underwoodii Trel., Mem. Nat. Acad. Sci. 11: 37. 1913.

27) A. portoricensis Trel., Mem. Nat. Acad. Sci. 11: 38. 1913.

28) A. cajalbanensis A. Álvarez, Revista Jard. Bot. Nac. Cuba 1(2-3): 33-39. 1980.

Habitan en las Antillas Mayores, Islas Cayman, Islas Vírgenes y St. Croix, en lugares abiertos y rocosos, desde el nivel del mar hasta casi los 2000 metros, prefieren los suelos calizos, pero también se presentan sobre suelos derivados de rocas ígneas y metamórficas.

Los estudios en esta sección han sido muy escasos a pesar de su alta incidencia en los diferentes tipos de vegetación en estas islas. Hummelinck $(1984,1985)$ analizó las especies de las Islas Cayman. Adams (1972) las especies de Jamaica y Álvarez (1980b) agregó una especie a la lista de las cubanas.

Las diferencias entre las especies de La Española representadas por $A$. antillarum (A. brevipetala, A. brevispina y $A$. intermixta) son muy sutiles y merecen una revisión pues parece tratarse de una sóla especie con amplia variación y distribución. Presumiblemente $A$. willdingii pueda incluirse en este grupo de especies.

En Jamaica los diferentes taxa parecen tener variaciones altitudinales y edáficas de la especie principal de esta isla, A. sobolifera, que también habita en las Islas Cayman.

En Cuba crecen varias especies que parecen variar de acuerdo al suelo y al propio aislamiento dentro de las regiones donde se desarrollan y que pudieran quizá someterse a la sinonimia de $A$. offoyana, nombre más viejo de la especie que crece en la costa norte de casi toda Cuba y que fue tomada por Trelease como A. legrelliana y así reportada por León (1946).

\section{Sección Caribaeae}

Plantas grandes, no cespitosas, no acaulescentes. Hojas de $100-200 \times 15-20 \mathrm{~cm}$, numerosas, algo flexuosas, acanaladas, verdes; espina corta, decurrente; dientes diminutos muy próximos. Inflorescencias grandes, panículas oblongas desde la mitad superior, ramas ascendentes. Flores de 45-70 $\mathrm{mm}$, amarillas. Cápsulas oblongas, poco frecuentes. Fuertemente bulbíferas.

29) A. karatto Miller, Gard. Dict. ed.8. 1768.

A. oducta Trel., Mem. Nat. Acad. Sci. 11: 25-26. 1913.

30) A. schevermaniana Trel., Mem. Nat. Acad. Sci. 11: 25. 1913.

31) A. vangrolae Trel., Mem. Nat. Acad. Sci. 11: 24. 1913.

A. nevidis Trel., Mem. Nat. Acad. Sci. 11: 24. 1913.

32) A. dussiana Trel., Mem. Nat. Acad. Sci. 11: 26-27. 1913.

A. montserratensis Trel., Mem. Nat Acad. Sci. 11: 24. 1913. 
33) A. caribaeicola Trel., Mem. Nat. Acad. Sci. 11: 27. 1913. A. medioxima Trel., Mem. Nat. Acad. Sci. 11: 24. 1913. A. unguiculata Trel., Mem. Nat. Acad. Sci 11:29. 1913. A. ventu-versa Trel., Mem. Nat. Acad. Sci 11: 29. 1913. 34) A. grenadina Trel., Mem. Nat. Acad. Sci. 11: 25. 1913. 35) A. barbadensis Trel., Mem. Nat. Acad. Sci 11: 28-28. 1913. 36) A. tranquera Trel., Mem. Nat. Acad. Sci. 11: 26. 1913.

Habitan en las Antillas Menores desde Anguilla hasta Grenada, sobre suelos calizos o volcánicos en lugares abiertos y escarpados.

Trelease (1913) practicamente describió una especie por una a tres islas de este gran archipiélago, esta concepción no fue aceptada por Howard (1979), quien las redujo a solo siete. Hummelinck, $(1986,1987)$, sometió a A. eggersiana a la sinonimia de $A$. missionum, única especie de Antillanae que crece en las Antillas Menores ( St. Thomas, St. John, Tortola y St. Croix).

\section{Sección Viviparae}

Plantas medianas a pequeñas, a veces cespitosas y subcaulescentes. Hojas de $40-100 \times 12-20 \mathrm{~cm}$, anchamente lanceoladas, cóncavas, verdes a veces grisáceas. Inflorescencias moderadas, panículas oblongas mayormente sobre la mitad superior. Flores de $45-55 \mathrm{~mm}$, amarillas, agrupadas al final de las ramas. Cápsulas oblongas. Fuertemente bulbíferas.

37) A. vivipara L., Sp. Pl. I: 323. 1753.

A. vicina Trel., Mem. Nat. Acad. Sci. 11: 19. 1913. 38) A. arubensis Hummelinck, Rec. Trav. Bot. Néerl. 33: 234. 1936.

39) A. rutteniae Hummelinck, Rec. Trav. Bot Néerl. 33: 238. 1936.

40) A. boldinghiana Trel., Mem. Nat. Acad. Sci. 11: 21. 1913. 41) A. cocui Trel., Mem. Nat. Acad. Sci. 11: 19. 1913.

42) A. evadens Trel., Mem. Nat. Acad. Sci. 11: 20.1913.

43) A. petiolata Trel., Mem. Nat. Acad. Sci. 11: 20. 1913.

Habitan en Trinidad, Aruba, Curazao, Bonaire y otras islas frente a las costa venezolana y en tierra firme de la región norteña costera y subcostera venezolano-colombiana.

Hummelinck $(1936,1938)$ realizó colectas y comparó las especies y localidades y no sólo agregó dos nuevas especies sino tambien variedades y subespecies a las ya conocidas, reconociendo que los límites entre ellas pudieran ser discutibles.

\section{ESPECIES DE AGAVE NATURALIZADAS O CULTIVADAS EN LAS ANTILLAS.}

La extracción de fibras y el uso como ornamentales permitió la introducción y en muchos casos naturalización de varias especies de Agave, entre las mas notorias se encuentran:

\section{A. fourcroydes Lem., Ill. Hort. 11:65. 1864.}

Esta especie conocida como henequén es abundante en Cuba y Haití, donde se establece en áreas marginales después de abandonar los cultivos. Se reproduce perfecta- mente por sus propios hijuelos, aunque no son raros los casos de producción de frutos con semillas viables.

A. sisalana Perrine, Congr. Doc. 554:8, 9, 16, 47, 60, 86, Sen. Doc. 300:36, 105, 140, (2nd. Session 25 Cong., USA), 1838.

Reportada por Trelease (1913) y Hummelinck (1986, 1987) para las Islas Cayman, Bahamas, Barbados y St. Croix.

A. angustifolia Haw., Syn. Pl. Succul. 72, 1812; 78. 1819.

Reportada por Gillis (1976) y Howard (1979) para Bahamas, Islas Cayman y St. Vincent.

Entre las especies ornamentales más frecuentes, pero apenas naturalizadas se encuentran:

A. angustifolia Haw. var. marginata Hort.

A. americana L., Sp. Pl. I:461. 1753.

A. americana L. var. expansa (Jacobi) Gentry, US Dept. Agr. Agricultural Handbook 399:80. 1972.

A. americana L. var. marginata Trel., in Bailey, Stand. Cycl. Hort. 1:235. 1866.

A. desmettiana Jacobi, Hamburg. Gart. u. B1.Zeit. 22:217. 1866.

A. franzosini Baker, Kew Bull. Miss. Inf. 3. 1892.

\section{AGRADECIMIENTOS}

Al M.en C. Abisaí García-Mendoza por facilitarme una parte de la literatura consultada y por su interés en que este artículo fuera publicado. Al Dr. Luis Eguiarte y Dra. Raquel Galván por la revisión del artículo. Al dibujante Felipe Villegas por hacer las figuras seis y siete.

\section{LITERATURA CITADA}

Adams CD. 1972. Flowering Plants of Jamaica. University of the West Indies, Kingston.

Álvarez A. 1979. Análisis de la epidermis foliar de Agave L. (Sec. Antillares-Cuba Occidental). Wissenschaft Zentralicher Friederich Schiller-Universität Jena, Mathematik-Naturwissenschaft 28: 665-674.

Álvarez A. 1980a. Agave jarucoensis A. Alvarez: Una nueva especie de Cuba Occidental. Revista del Jardín Botánico Nacional de Cuba 1: 5-11.

Álvarez A. 1980b. Agave cajalbanensis: Una nueva especie de Cuba Occidental. Revista del Jardín Botánico Nacional de Cuba 1: 33-40.

Álvarez A. 1985. Los agaves de Cuba Occidental. Revista del Jardín Botánico Nacional de Cuba 5: 3-16.

Álvarez A. 1989. Distribución geográfica y posible origen de las Agavaceae. Revista del Jardín Botánico Nacional de Cuba 10: 25-36.

Álvarez A. 1990. El complejo estomático en la familia Agavaceae. II. Epidermis adulta. Feddes Reppertorium 100: 113-134.

Berger A. 1915. Die Agaven. Verlag, Jena.

Blunden G, Jewers K. 1973. The comparative leaf anatomy of Agave, Beschorneria, Doryanthes and Furcraea species (Agavaceae: Agaveae). Botanical Journal of the Linnean Society 66: 157-179.

García-Mendoza A. 1995. Riqueza y endemismos de la familia Agavaceae en México. En: Linares E, Dávila P, Chiang F, Bye R, Elias T, 


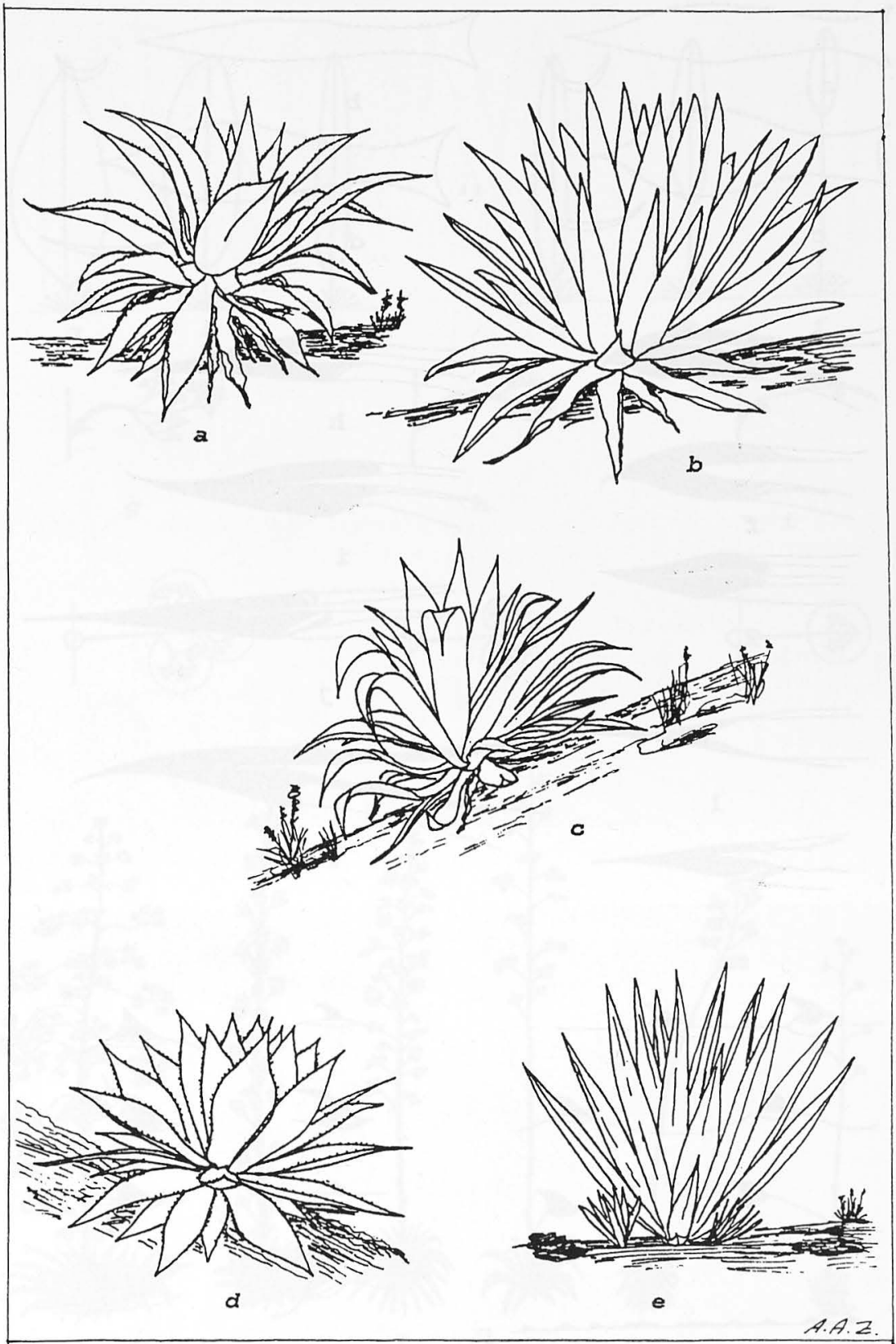

FIgura 1. Hábito de las plantas. a, Agave vivipara (X/17). b, A. karatto (X/25). c, A. sobolifera (X/30). d, A. tubulata (X/18). e, A. inaguensis (X/12). 


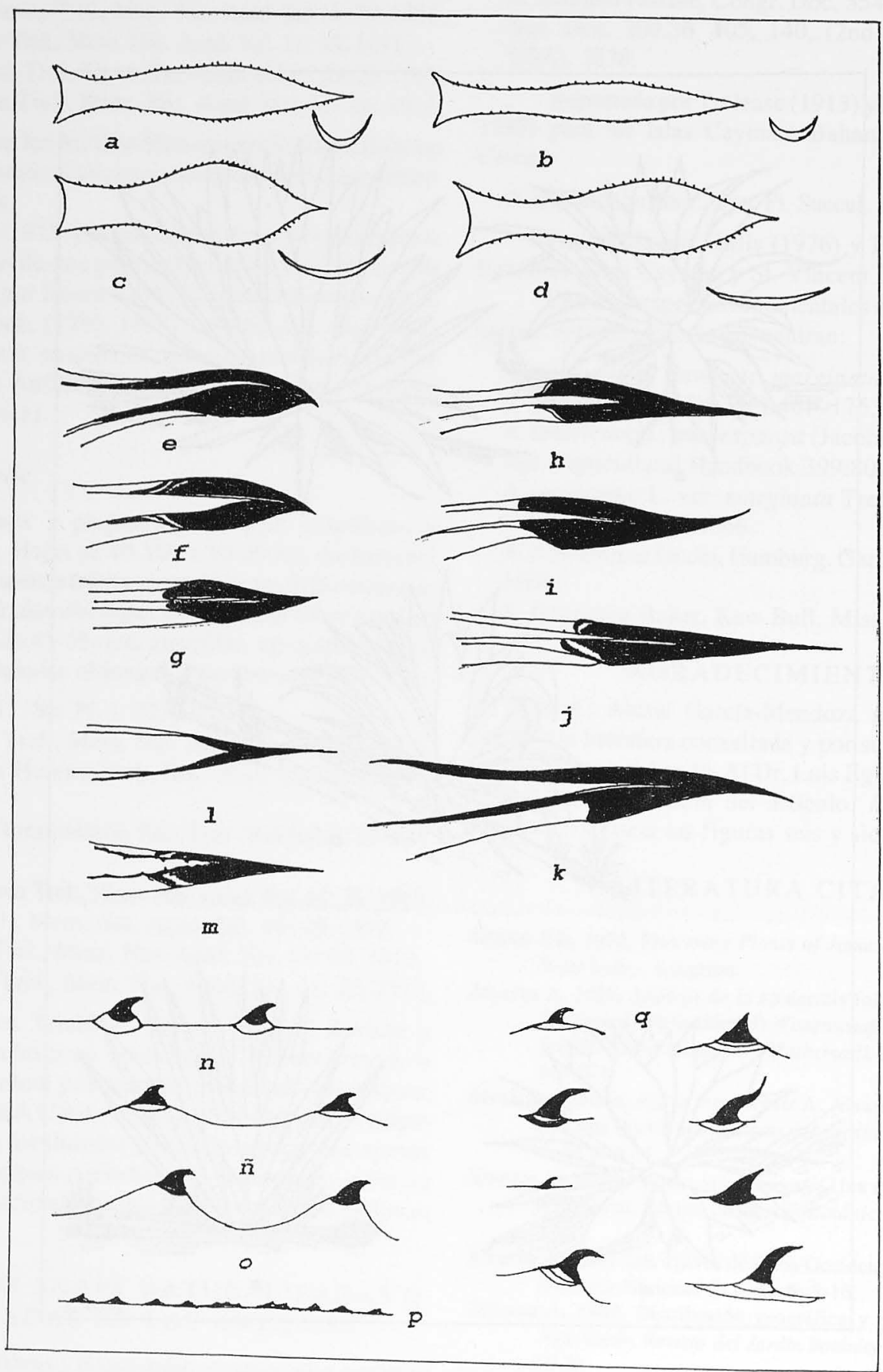

Figura 2. Caracteres foliares. Forma de las hojas: a, lanceoladas. b, estrechamente lanceoladas. c-d, anchamente lanceoladas. Concavidad foliar: a-b, cóncavas (acanaladas). c, semicóncavas, d, casi planas. Forma de la espina terminal: e-g, obtusas con el canalículo muy largo. h-k, agudas con el canalículo moderado. $1-\mathrm{m}$, aciculares con el canalículo breve. Forma de los dientes del margen y su separación: n-o, bien desarrollados y subdistantes. p, diminutos y muy próximos. q, diferentes formas de dientes. 


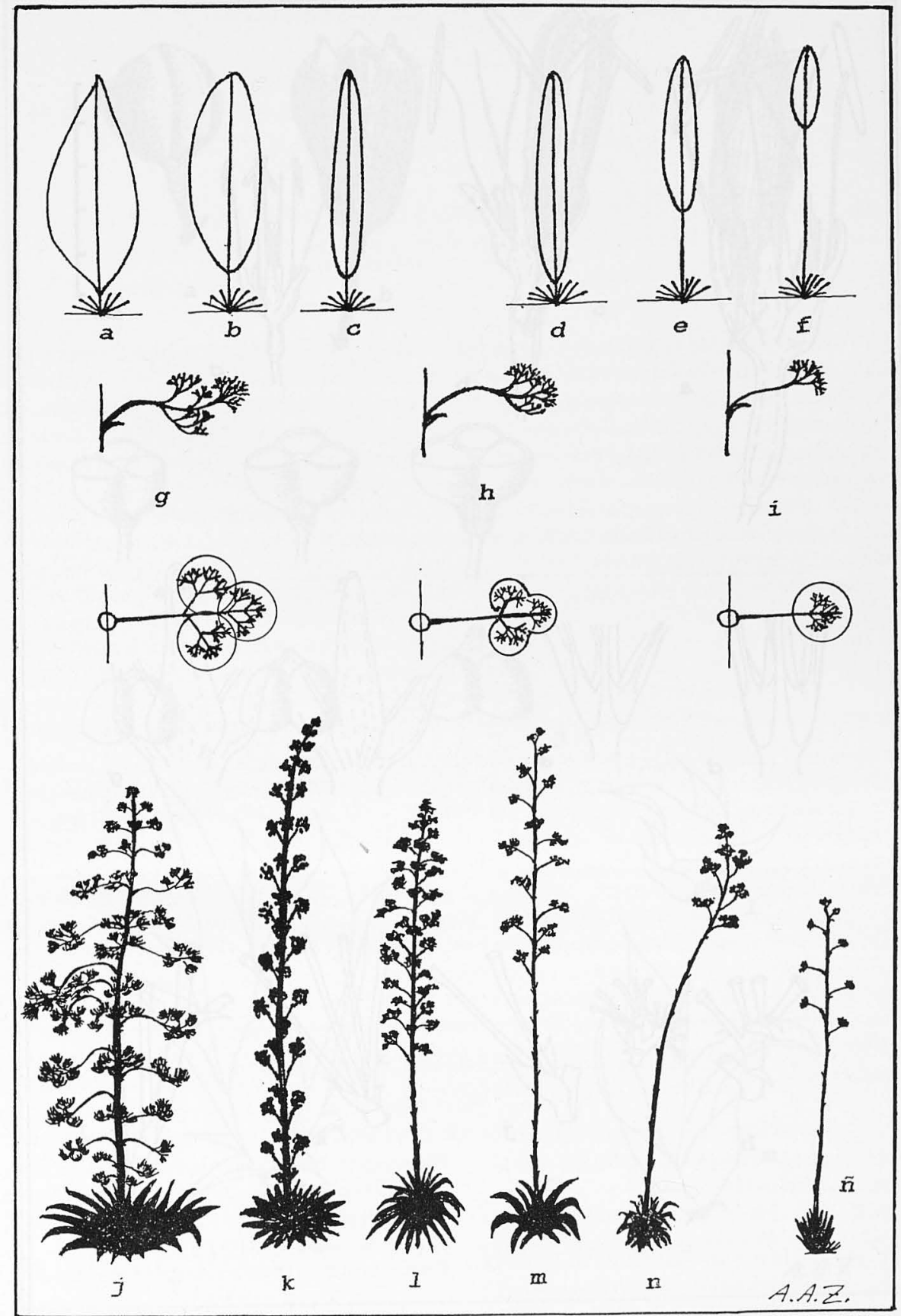

Figura 3. Caracteres de la inflorescencia. Tipo de panícula: a, oblongo piramidal. b, oblonga. c, estrechamente oblonga a fusiforme. Desarrollo relativo del pedúnculo floral: d, breve o casi nulo. e, moderado. f, alargado. Formas de las ramas de la panícula: g, bien desarrolladas y tripartidas. h, algo compactas y trilobadas. i, muy compactas y unilobadas. Siluetas de algunas especies antillanas de Agave: j, A. offoyana (X/100). k, A. brittoniana (X/40). 1, A. portoricensis (X/100). m, A. bahamana (X/100). n, A. vivipara (X/40). ñ, A. nashii(X/30). 


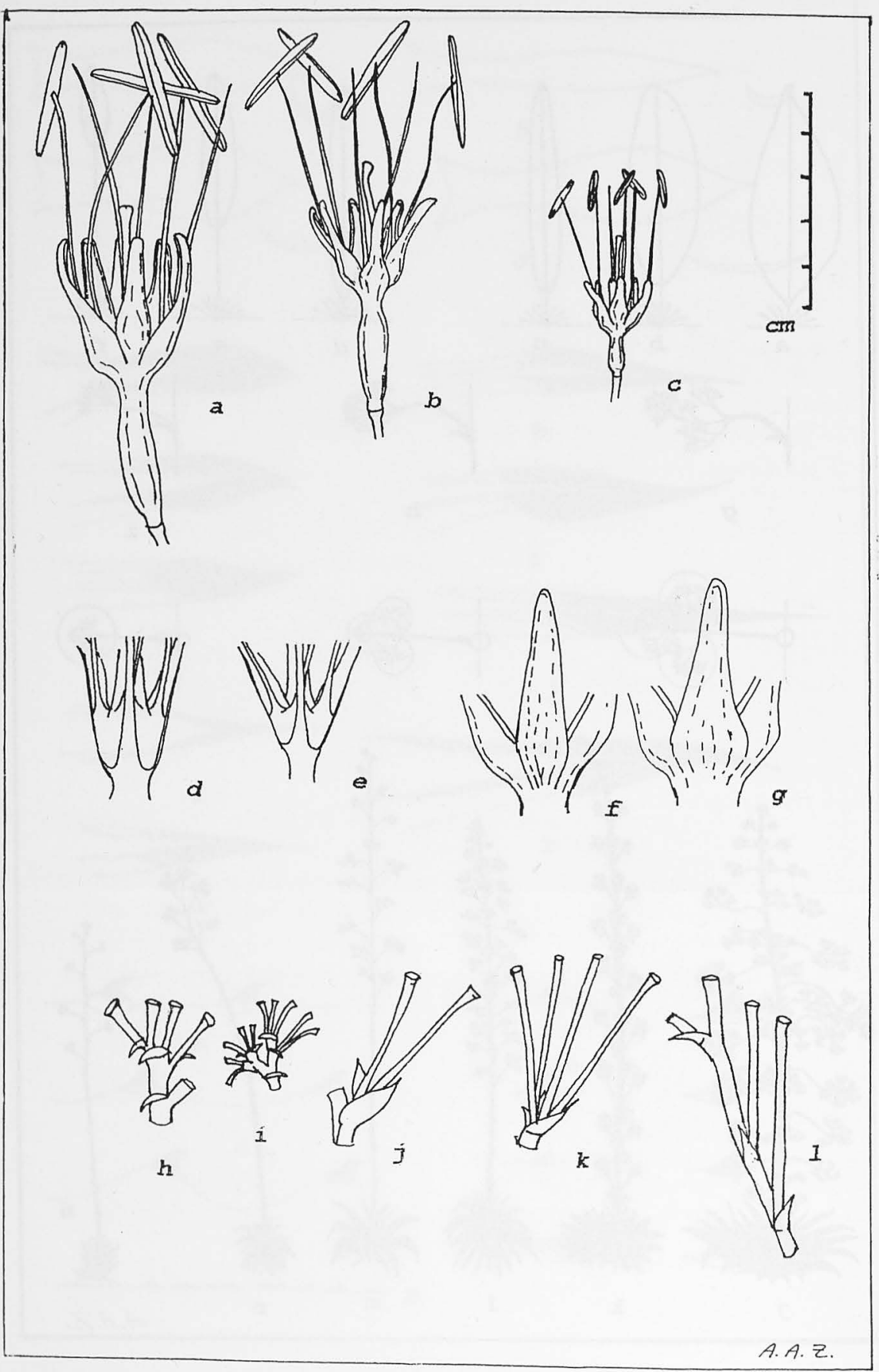

Figura 4. Caracteres de las flores: a, flores grandes de A. offoyana. b, flores medianas de A. sobolifera. c, flores pequeñas de $A$. albescens. d, tubo perigonal profundo de $A$. tubulata ssp. tubulata. e, tubo perigonal breve de $A$. tubulata ssp. brevituba. f, tépalos estrechos de A. grisea. g, tépalos anchos de A. karatto. h-1, formas de los pedicelos. 


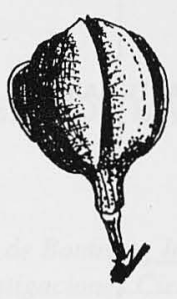

a
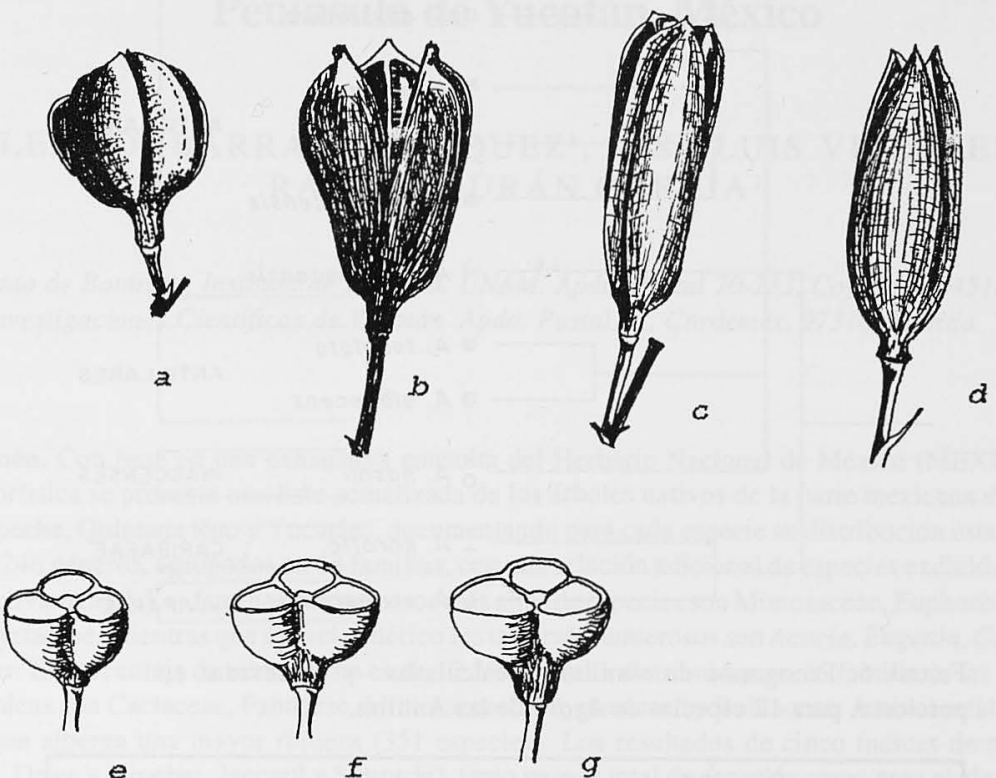

e

E

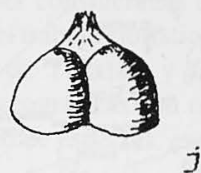

j
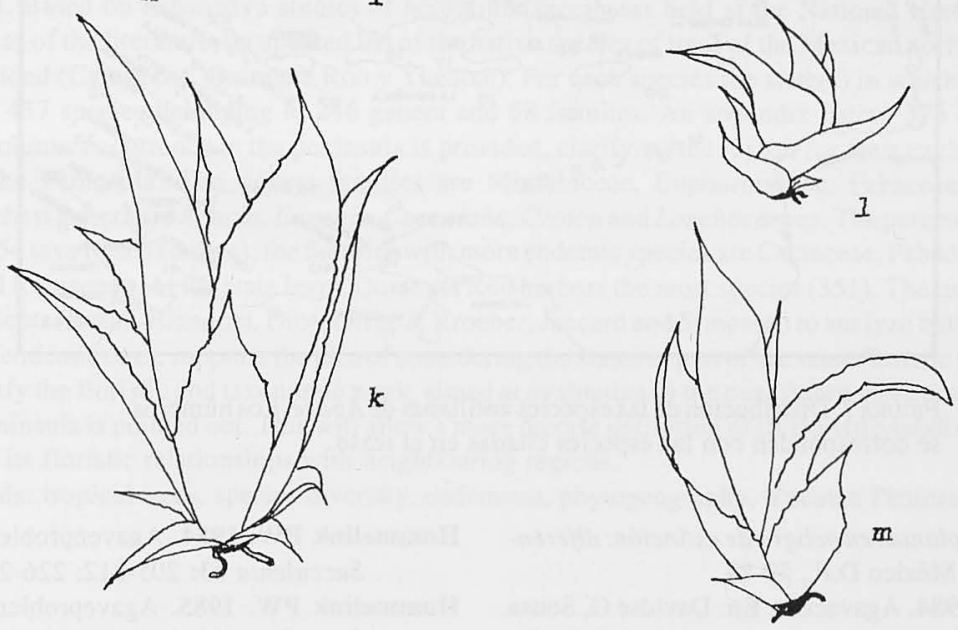

A. A.Z

Figura 5. Caracteres de los frutos y bulbilos: a, frutos globosos de $A$. portoricensis. b, frutos oblongos de $A$. sobolifera. c, frutos subcilíndricos de $A$. offoyana. d, frutos estrechamente oblongos de $A$. bahamana (A. indagatorum). Base de los frutos: e, corto.f, moderado. g, alargado. Apice de los frutos: h, corto. i, moderado. j, rostrado. Bulbilos: k, A. karatto. l, A.anomala. m, A. vivipara. 


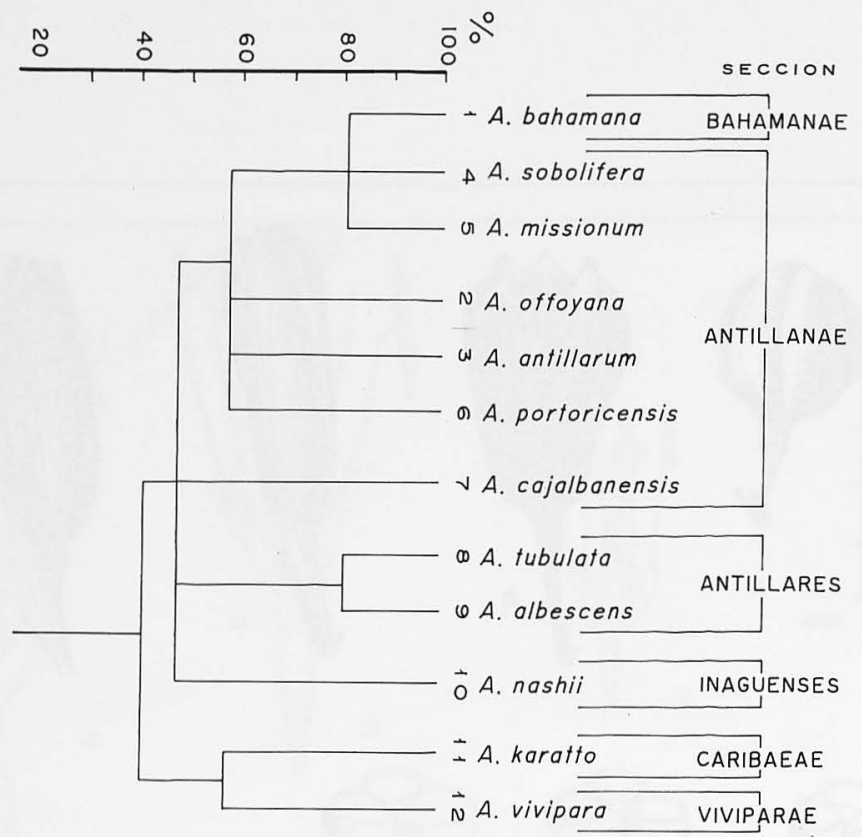

FIgura 6. Fenograma de similitudes calculadas y expresadas en porciento, para 12 especies de Agave de las Antillas.

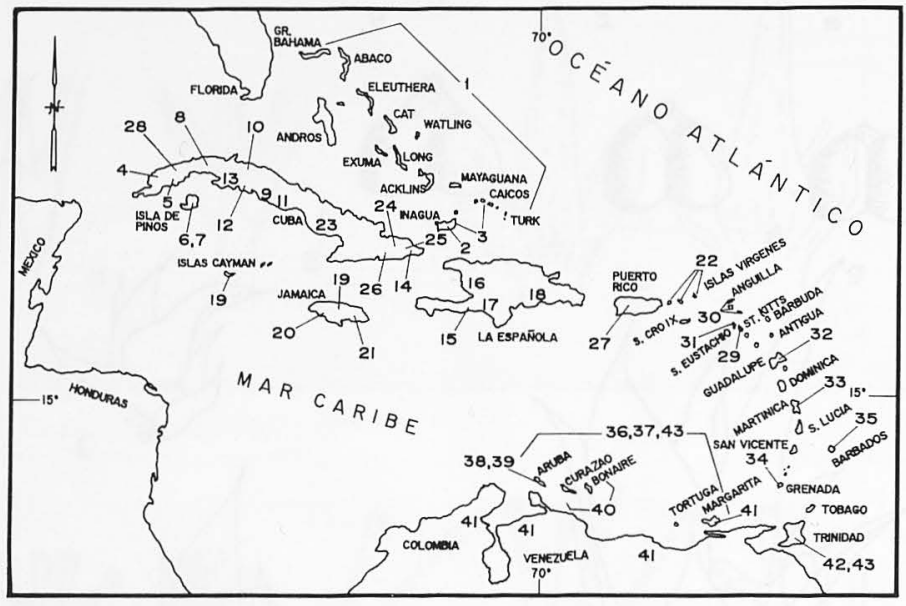

Figura 7. Distribución de las especies antillanas de Agave. Los números se corresponden con las especies citadas en el texto.

edrs. Conservación de plantas en peligro de extinción: diferentes enfoques. UNAM, México D.F., 59-83.

García-Mendoza A, Lott E. 1984. Agavaceae. En: Davidse G, Sousa M, Chater AO, edrs. Flora Mesoamericana. Vol. 6. UNAM, México D.F., 35-47.

Gentry HS. 1982. Agaves of Continental North America. University Arizona Press, Tucson.

Gillis, WT. 1976. Agave in the Bahama Islands. Phytologia 33: 78-81.

Howard RA. 1979. Flora of the Lesser Antilles, Leeward and Windward Islands. Thompson-Mills, Mass.

Hummelink PW. 1936. Notes on Agave in Aruba, Curacao, Bonaire and some parts of south american continent. Recueil des Travaux Botaniques Neerlandais. 33: 223-249.

Hummelink PW. 1938. Notes on Agave in the Netherlands West Indies and North Venezuela. Recueil des Travaux Botaniques Neerlandais. 35: 14-28.
Hummelink PW. 1984. Agavenproblemen op de Cayman eilanden. Succulenta 63: 205-212; 226-228; 259-263; 227-282.

Hummelink PW. 1985. Agaveproblemen Op de Cayman-eilanden. Succulenta 64: 9-11.

Hummelink PW.1986. Agavenproblemen Op de Bovenwindse Eilanden der Kleine Antillen (I-II). Succulenta 65: 165-174; 215-219.

Hummelink PW.1987. Agavenproblemen op de Bovenwindse Eilander der Kleiner Antillen (III-VIII). Succulenta 66: 10-13; 65-69; 127-139; 187-189; 205-211; 265-270.

Labat RP. 1722. Vollages aux Isles de L'Amerique. Casa de las Américas (1979), La Habana.

León H. 1946. Flora de Cuba I. Contribuciones Ocasionales Museo Historia Natural Colegio De La Salle 8: 316-319.

Trelease W. 1913. Agave in the West Indies. Memoirs of the National Academy of Sciences. 11: 1-55.

Trelease W. 1915. The Agaveae of Guatemala. Transactions of the Academy of Science of St. Louis 23: 129-147. 